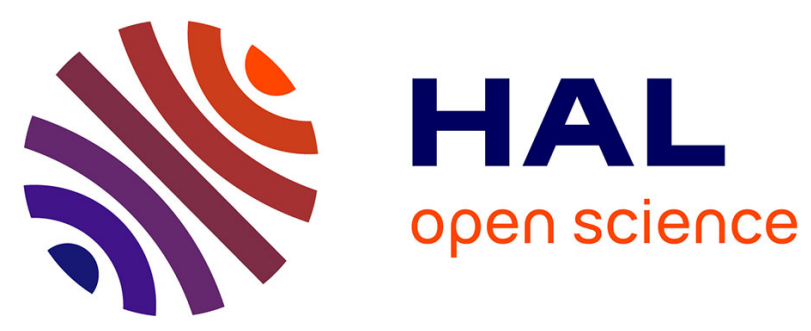

\title{
Polar mixture analysis by NMR under spin diffusion conditions in viscous sucrose solution and agarose gel
}

Pedro Lameiras, Simon Mougeolle, François Pedinielli, Jean-Marc Nuzillard

\section{To cite this version:}

Pedro Lameiras, Simon Mougeolle, François Pedinielli, Jean-Marc Nuzillard. Polar mixture analysis by NMR under spin diffusion conditions in viscous sucrose solution and agarose gel. Faraday Discussions, 2019, 218, pp.233-246. 10.1039/C8FD00226F . hal-03349854

\section{HAL Id: hal-03349854 https://hal.science/hal-03349854}

Submitted on 20 Sep 2021

HAL is a multi-disciplinary open access archive for the deposit and dissemination of scientific research documents, whether they are published or not. The documents may come from teaching and research institutions in France or abroad, or from public or private research centers.
L'archive ouverte pluridisciplinaire HAL, est destinée au dépôt et à la diffusion de documents scientifiques de niveau recherche, publiés ou non, émanant des établissements d'enseignement et de recherche français ou étrangers, des laboratoires publics ou privés. 


\title{
Journal Name
}

\section{ARTICLE}

\section{Polar Mixture Analysis by NMR under Spin Diffusion Conditions in Viscous Sucrose Solution and Agarose Gel}

Received 00th January 20xx, Accepted 00th January 20xx

DOI: $10.1039 / \times 0 \times x 00000 x$

www.rsc.org/

\author{
Pedro Lameiras, ${ }^{*}{ }^{\mathrm{a}}$ Simon Mougeolle ${ }^{\mathrm{a}}$, François Pedinielli ${ }^{\mathrm{a}}$ and Jean-Marc Nuzillard ${ }^{*} \boldsymbol{}^{\mathrm{a}}$
}

The use of two new viscous solvents, sucrose solution and agarose gel, is reported for the first time for giving access to the individual NMR spectra of polar and potentially bioactive compounds in mixture. Under viscous conditions, the tumbling rate of small and mid-sized molecules falls down in solution, so that the longitudinal cross-relaxation encourages the observation of spin diffusion. As a result, all the resonances of the ${ }^{1} \mathrm{H}$ nuclei within the same molecule tend to correlate together in a 2D NOESY spectrum, thus opening the way to mixture analysis. This work describes the individualization of four structurally close mixed dipeptides : Leu-Val, Leu-Tyr, Gly-Tyr and Ala-Tyr dissolved respectively in sucrose solution and agarose gel by means of spin diffusion in homonuclear selective 1D NOESY, selective 2D NOESY experiments and heteronuclear 2D HSQC-NOESY. Sucrose solution should be preferred to agarose gel for the investigation of mixtures made of small and flexible polar compounds due to its capability to give rise to more suitable viscous conditions mandatory for efficient spin diffusion even though agarose gel reveals the benefit of not offering intense residual proton solvent due to active

transverse

relaxation.

\section{Introduction}

The identification of organic molecules in mixture is a crucial issue in the vast majority of human activities related to chemistry (healthcare, energy, materials, etc.). Transformation processes that lead from natural resources, either from fossil carbon industry or biomass exploitation, to low-value products in large amounts or to high-value speciality products including pharmaceutical and cosmetic active ingredients, rarely produce chemically pure compounds. In the current state of knowledge, mixture analysis remains a necessity and to date addressing this issue by NMR spectroscopy has been explored in a reduced number of ways. i) Translational diffusion coefficient (D) characterizes molecules in solution and reflects their mobility. In theory, sub-spectra of the NMR spectrum of a mixture can be extracted using $D$ as discriminating factor, so as to reach the spectra of the individual mixture components. ${ }^{1-3}$ Nonetheless, D values are poorly discriminant, although experimental tricks enable an increase of the resolving power in special cases: introduction of various chromatographic solid phases in the sample, interaction of analytes with soluble polymers or lanthanide shift reagents, analyte inclusion in micelles. ${ }^{4-14}$ ii) Multi-quantum spectroscopy combined

\footnotetext{
a. Université de Reims Champagne-Ardenne, Institut de Chimie Moléculaire de Reims, CNRS UMR 7312, SFR CAP-Santé, BP 1039, 51687 Reims Cedex 02, France. E-mail: pedro.lameiras@univ-reims.fr, Tel :+33 326918228.

E-mail: jm.nuzillard@univ-reims.fr, Tel :+33 326918210

+ Contributed equally to the work.

Electronic Supplementary Information (ESI) available: [details of any supplementary information available should be included here]. See DOI: $10.1039 / \times 0 \times x 00000 x$
}

(or not) with broadband homonuclear decoupling, sparse sampling and pure shift data acquisition may be pertinent for individualizing molecules in a mixture. For example, a simple 2D TOCSY spectrum or series of 1D selective TOCSY spectra points out ${ }^{1} \mathrm{H}$ nuclei coupled together, which may turn out to be useful for molecules composed only of one single spin system. ${ }^{15-21}$ iii) Viscous solvents (or solvent blends) under specific operating conditions lower the tumbling rate of small and mid-sized molecules in solution since the value of the molecular overall correlation time $\tau_{c}$ depends upon the medium viscosity according to the microviscosity theory of Gierer and Wirtz. 22 The longitudinal cross-relaxation regime thus favours the detection of spin diffusion. Consequently, the molecules present a negative nuclear Overhauser effect (NOE) regime and their resonances can be grouped according to their ability to share magnetization through intramolecular diffusion of spin magnetization. All the resonances of the ${ }^{1} \mathrm{H}$ nuclei within the same molecule tend to correlate together in a 2D NOESY spectrum, thus giving access to the individual ${ }^{1} \mathrm{H}$ NMR spectra of the mixture components. The original idea was implemented in $1981^{23}$ by means of a perfluorinated polymer solvent and was considered again in 2008; ${ }^{24}$ the use of supercooled water for tailoring the spin dynamics of small metabolites was published in 2012 by the same author. ${ }^{25}$ Our team reported for the first time, respectively in 2011, 2016 and 2017, the use of glycerol and glycerol carbonate, of DMSO/glycerol and of DMSO/water binary solvents as viscous media for ${ }^{1} \mathrm{H}$ and ${ }^{19} \mathrm{~F}$ spin diffusion promotion. ${ }^{26-28}$

The present work focusses on the assessment of sucrose solution and agarose gel in the individual NMR characterization of four polar structurally close dipeptides: Leu-Val, Leu-Tyr, Gly-Tyr 
and Ala-Tyr within a single mixture by means of spin diffusion in homonuclear selective 1D NOESY, selective 2D NOESY experiments and heteronuclear 2D HSQC-NOESY.

Dissolving "table sugar" in water yields a viscous solution that enables us to take profit from spin diffusion from room temperature to a below temperature due to the overall decrease in the tumbling rate of molecules in solution. For instance, the viscosity of a sucrose/water (1:1, w/w) blend is around $27 \mathrm{cP}$ at 283 K. ${ }^{29,30}$ In comparison, that of water at the same temperature is $1.307 \mathrm{CP} .{ }^{31}$ At room temperature, the viscosity of the same sucrose/water blend is sufficiently low, so that NMR samples may be prepared and transferred into an NMR tube without any difficulty, in contrast to highly viscous solvents such as glycerol ( $\eta=$ $934 \mathrm{cP}$ at $298 \mathrm{~K})^{32}$ and glycerol carbonate $(\eta=85.4 \mathrm{cP}$ at $298 \mathrm{~K}){ }^{32}$ Adding sucrose to water (deuteurated or not) also opens the way of working from room to sub-zero temperature, ${ }^{30}$ which is especially suitable for thermally unstable molecules such as bioactive molecules. Spin diffusion may thus happen on a wide range of temperatures, from $243 \mathrm{~K}^{30}$ to room temperature and even higher. In addition, the sample preparation cost is very low since the "table sugar" is a widespread staple. However, the major experimental pitfall is strong residual proton signals of non-deuterated sucrose that obliges its mandatory elimination. Mid-sized molecules will only necessitate a low amount of sucrose in water whereas smaller molecule will require more sucrose for driving spin diffusion under temperature control from room temperature to sub-zero temperature.

Agarose gel is commonly used for the separation of biological molecules by electrophoresis even if it was already proposed as a confinement medium for investigating the molecular confinement effect on the conformational dynamics of apomyoglobin. ${ }^{33}$ The main problem in the sample preparation is the addition of molecules of interest to the agarose powder previously dissolved in water at high temperature ( $363 \mathrm{~K}$ for melting regular agarose and at around $338 \mathrm{~K}$ for low-melting agarose). Peptide or protein solutions may suffer from the high temperature exposition preventing NMR structural and conformational studies and premature gelation might occur. In practice, the heat time only takes a few minutes, thus the sample preparation is compatible with quite small molecules reasonably heat resistant such as nucleic acids, peptides or saccharides. In addition, NMR signal line sharpness can dramatically be altered in the case of inhomogeneous samples due to partially unmelted agarose or incorporation of air bubbles. The solution consists to melt the sample again directly inside the NMR tube, then to mix thoroughly it and chill again at ambient temperature. Interestingly, agarose is non-interactive with most biomolecules and organic compounds so that DNA, RNA and protein can be recovered from agarose gels. ${ }^{34,}{ }^{35}$ Its freezing point is reported from $260 \mathrm{~K}$ and $264 \mathrm{~K}$ routinely reached respectively with $\mathrm{H}_{2} \mathrm{O}$ - and $\mathrm{D}_{2} \mathrm{O}$-based $1 \%$ agarose gels ${ }^{36}$ limiting the temperature range for which spin diffusion may occur compared to sucrose solution. Hence, agarose gel will be more dedicated to the study of rigid small or medium-sized molecules in mixture presenting higher correlation times than very flexible small molecules. Remarkably, due to the rigidity of the agarose gel thus the too active transverse relaxation, all resulting proton and carbon resonances are too broad to be detected in NMR spectroscopy. ${ }^{36,37}$ As a result, only the residual proton signal of water has to be removed compared to numerous proton signals of non-deuterated sucrose.

The criterion for optimal temperature selection is a compromise between overall spectral resolution and intensity of NOESY cross peaks between nuclei that are not close enough to show a NOE signal in a low viscosity medium. A temperature reduction enhances spin diffusion but also reduces peak height through line broadening caused by a more efficient transverse relaxation process. Sample cooling is therefore required if the NOESY spectrum shows positive NOE responses (diagonal and off-diagonal peaks of opposite signs).

Depending on the complexity of the mixtures, the analysis of ${ }^{1} \mathrm{H}$ NMR spectra may become intractable due to the overlapping of ${ }^{1} \mathrm{H}$ resonances. A usual remedy to this issue consists in the spreading of the spectroscopic information along a second axis that encodes chemical shifts of nuclei other than ${ }^{1} \mathrm{H}$ such as ${ }^{13} \mathrm{C}$ or ${ }^{15} \mathrm{~N} .{ }^{27,}{ }^{28}$ This approach to mixture analysis is exemplified in sucrose solution by 1D and $2 \mathrm{D}{ }^{1} \mathrm{H}_{-}{ }^{15} \mathrm{~N}$ and ${ }^{1} \mathrm{H}-{ }^{13} \mathrm{C}$ HSQC-NOESY spectra providing ${ }^{1} \mathrm{H} /{ }^{13} \mathrm{C}$ and ${ }^{1} \mathrm{H} /{ }^{15} \mathrm{~N}$ chemical shift lists for the mixture components.

\section{Results and discussion}

\section{Leu-Val, Leu-Tyr, Gly-Tyr and Ala-Tyr mixture in sucrose solution.}

These four dipeptides in pure water solution do not reveal any differentiation based on their translational diffusion behaviour, due to their similar molecular weight and shape (Figure S-1 in the Electronic Supplementary Information (ESI)). This observation encouraged us to investigate the dipeptide mixture dissolved in viscous solution sucrose by considering the resolving power of homo- and heteronuclear NOESY-based spin diffusion experiments for offering pertinent alternatives to DOSY experiments. The main experimental drawback of our approach was the mandatory elimination of the strong ${ }^{1} \mathrm{H}$ signals of sucrose and water (see Figure 1a) for avoiding to excessively obscure solute signals since deuterated sucrose would be too expensive to produce and deuterated water would give rise to the chemical exchange between the deuterium nuclei of the latter and the amide proton of the dipeptides mixture. The elimination of these signals can be achieved by means of selective excitation and detection pulses when included in an excitation sculpting sequence. ${ }^{38}$ The selective pulses invert the equilibrium magnetization of the nuclei of interest and leave untouched the one of the solvent nuclei. For this purpose, resonance inversion in the two frequency bands on either side of the solvent signal was successfully realized by means of two consecutive band selective pulses. The spectrum in Figure $1 \mathrm{~b}$ proves the quality of solvent suppression that was achieved by band selective detection (see pulse sequence in Figure 1e).

Since the temperature is an important parameter in spin diffusion experiments because it is directly related to solvent viscosity and as a result to overall rotational correlation times $\tau_{\mathrm{C}},{ }^{22,}$ ${ }^{26}$ we have determined the optimal temperature at which NOESY 
cross peaks were positive (negative NOE enhancements, slow motion regime), well-resolved, and as intense as possible between nuclei that were not supposed to be close enough to present a NOE in low viscous medium. The optimized temperature of $283 \mathrm{~K}$ has been defined by means of band-selective detection NOESY experiments (see Figure 2a, Figures S-2: amide proton region NOESY spectra at 298, 288, 283, 278 and $273 \mathrm{~K}$ and S-3: full NOESY spectrum at $283 \mathrm{~K}$ in ESI). We have clearly observed that the use of viscous sucrose solution makes possible full intramolecular magnetization transfer through spin diffusion, observed over distances of $>14 \AA$ within each very small and flexible dipeptide. In comparison, the NOESY spectrum recorded in water at $298 \mathrm{~K}$ displays less numerous NOE cross peaks of opposite sign (positive NOE enhancements, fast motion regime, see Figure $2 b$, full NOESY spectrum in Figure S-4 in ESI). Hence, under viscous conditions, the grouping of proton resonances together is accessible allowing the individualization of the mixture components since the chemical shift pattern of each dipeptide is predictable. The individualization of the four dipeptides in water would have necessitated the concomitant use of NOESY and TOCSY (and COSY) experiments, according the common resonance assignment strategy. ${ }^{39}$

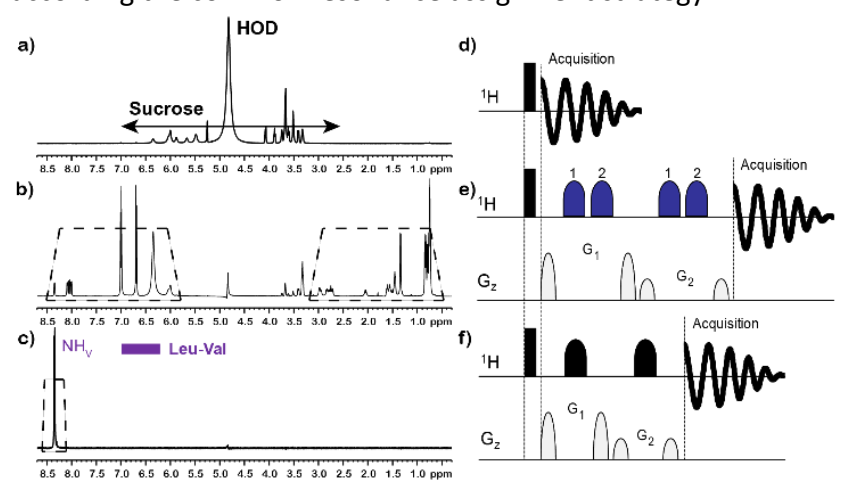

Fig. 1. $1 \mathrm{D}{ }^{1} \mathrm{H}$ spectra (8 scans), at $600 \mathrm{MHz}\left({ }^{1} \mathrm{H}\right)$, and corresponding NMR pulse sequence of the dipeptide test mixture in sucrose solution (a, b, c, $283 \mathrm{~K}$ ). G1:G2 = 70:30. The FIDs (32k points, spectral width $=6010 \mathrm{~Hz}$ ) were processed with $\mathrm{LB}=0.3 \mathrm{~Hz}$ and zerofilled to $32 \mathrm{k}$ points. a, d) Non-selective excitation and detection. $b$, e) Selective detection of two resonance bands. The $3 \mathrm{~ms}$ I-BURP-2 pulses cover $1560 \mathrm{~Hz}$ (dotted trapezium). The "1" and "2" labels respectively indicate their application to the high and low chemical shift regions. c, f) Selective excitation of the valine amide proton doublet of Leu-Val (dotted trapezium) using a $10 \mathrm{~ms}, 1 \%$ truncated, $180^{\circ}$ Gaussian pulse.

For analytical reasons, it appeared relevant to collect supplementary structural information of mixture components by detecting $\mathrm{H} \alpha$ proton resonances in $\mathrm{F}_{1}$ during signal acquisition, that was not the case by means of band selective transient NOESY experiments. By the way, we implemented a 1D selective NOESY pulse sequence composed of a double pulse field gradient block for the multiplet selective excitation ${ }^{38,40}$ of the resonance of interest followed by a mixing time including two wideband adiabatic inversion pulses flanked with gradient pulses so as to avoid the reintroduction of strong sucrose and water solvent signals during the mixing time that arise from longitudinal relaxation. Figure 3 undoubtedly proves that all dipeptides are differentiated by spin diffusion in sucrose solution by means of an appropriate set of selectively excited proton resonances. Indeed, the selective excitation of the $\mathrm{NH}$ amide proton, at $\delta 8.35$ in sucrose solution, shows a magnetization exchange exclusively with the protons of the Leu-Val dipeptide because the tyrosine $\mathrm{H} \delta / \mathrm{H} \varepsilon$ proton resonances do not appear in the 1D NOESY spectra (see Figure 3a). The selective excitation of the side chain $\mathrm{H} \delta$ and $\mathrm{H} y$ protons (between 0.7 and $0.9 \mathrm{ppm}$ ) displays a magnetization exchange with all protons of the two Leu-Val and Leu-Tyr dipeptides (see Figure $3 b$ ). By comparison with the 1D NOESY spectra in Figures $3 a$ and $3 b$, a complete proton assignment of Leu-Tyr is possible. The selective excitation of the aromatic $\mathrm{H} \delta / \mathrm{H} \varepsilon$ protons of Leu-Tyr, Gly-Tyr and Ala-Tyr reveals all the proton resonances of Leu-Tyr, Gly-Tyr and Ala-Tyr (see Figure 3c). One of the three tyrosine H $\alpha$ protons at $\delta$ 4.14 has been selectively excited in order to differentiate all proton resonances from Gly-Tyr and Ala-Tyr (see Figure 3d). Figure 3d clearly shows the transfer of the tyrosine $\mathrm{H} \alpha$ magnetization over all protons of Gly-Tyr because of the absence of the side chain proton $\left(H \beta_{A} / \beta_{L} / \gamma_{L} / \delta_{L}\right)$ resonances of Leu-Tyr and Ala-Tyr.

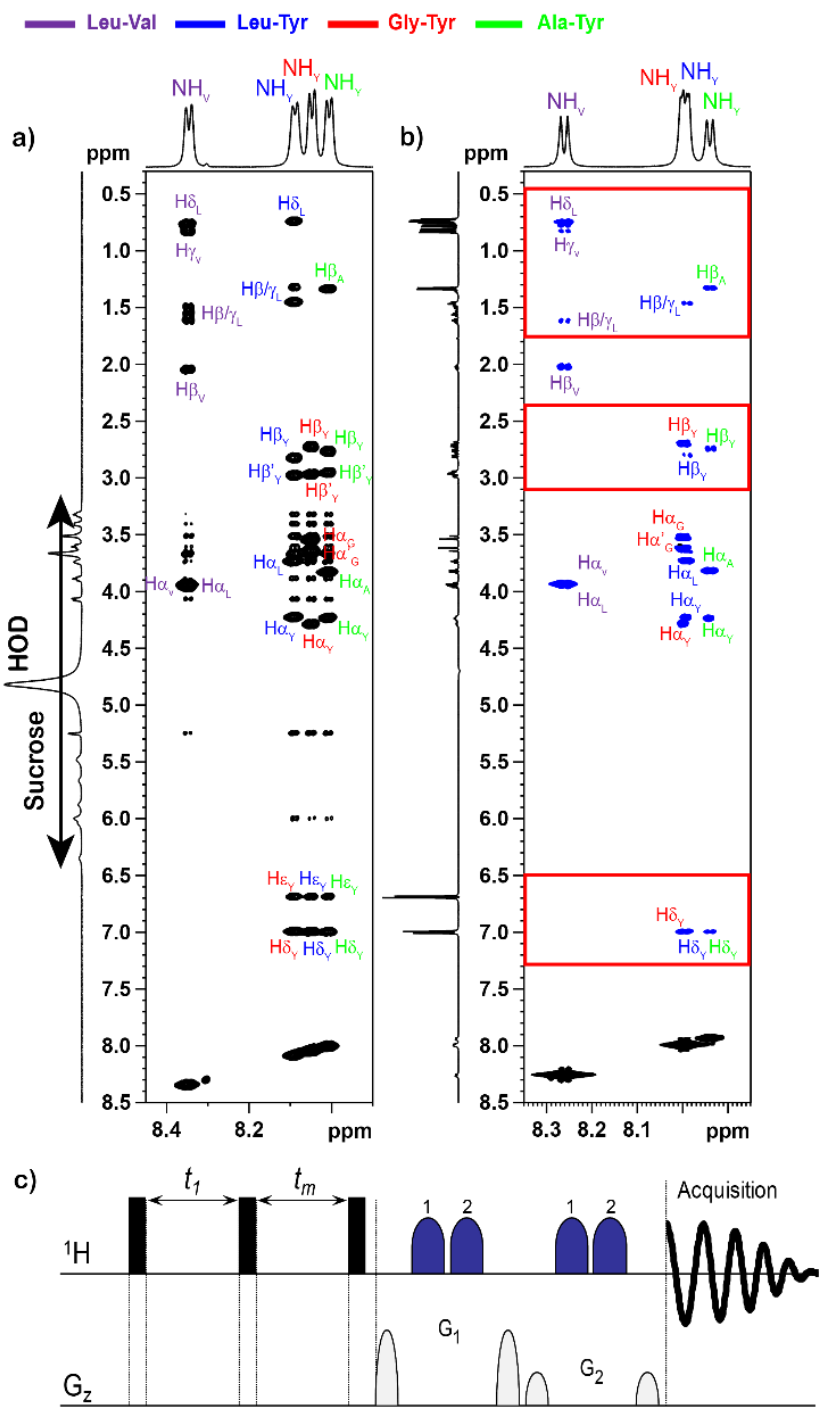


Fig. 2. a) Amide proton region of band-selective detection $2 D$ NOESY spectrum of the dipeptide test mixture $(10 \mathrm{mM})$, mixing time $\left(t_{m}\right)=0.5 \mathrm{~s}$, at $600 \mathrm{MHz}\left({ }^{1} \mathrm{H}\right)$ using the pulse sequence in part $\mathrm{c}$, dissolved in sucrose solution, at $283 \mathrm{~K}$. b) Amide proton region of 2D NOESY spectrum of the same dipeptide test mixture (10 mM), $t_{m}$ $=0.5 \mathrm{~s}$, at $600 \mathrm{MHz}\left({ }^{1} \mathrm{H}\right)$, using noesyesgpph pulse sequence, dissolved in $\mathrm{H}_{2} \mathrm{O} / \mathrm{D}_{2} \mathrm{O}(9: 1, \mathrm{v} / \mathrm{v})$, at $298 \mathrm{~K}$. The red frames correspond to spectral regions of interest in which water as solvent has a major effect on the number and sign of observable NOESY cross peaks.

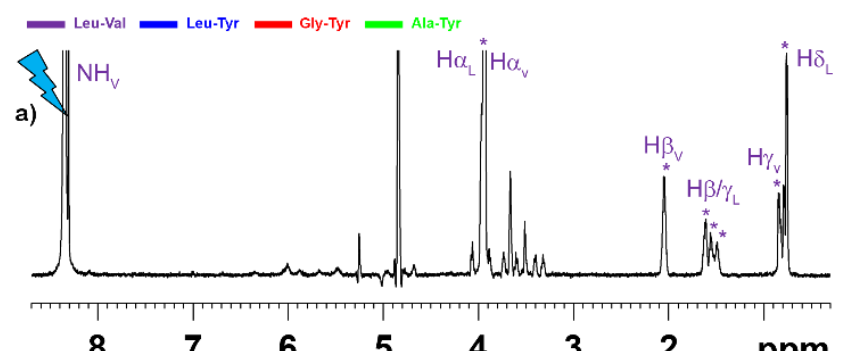

b)
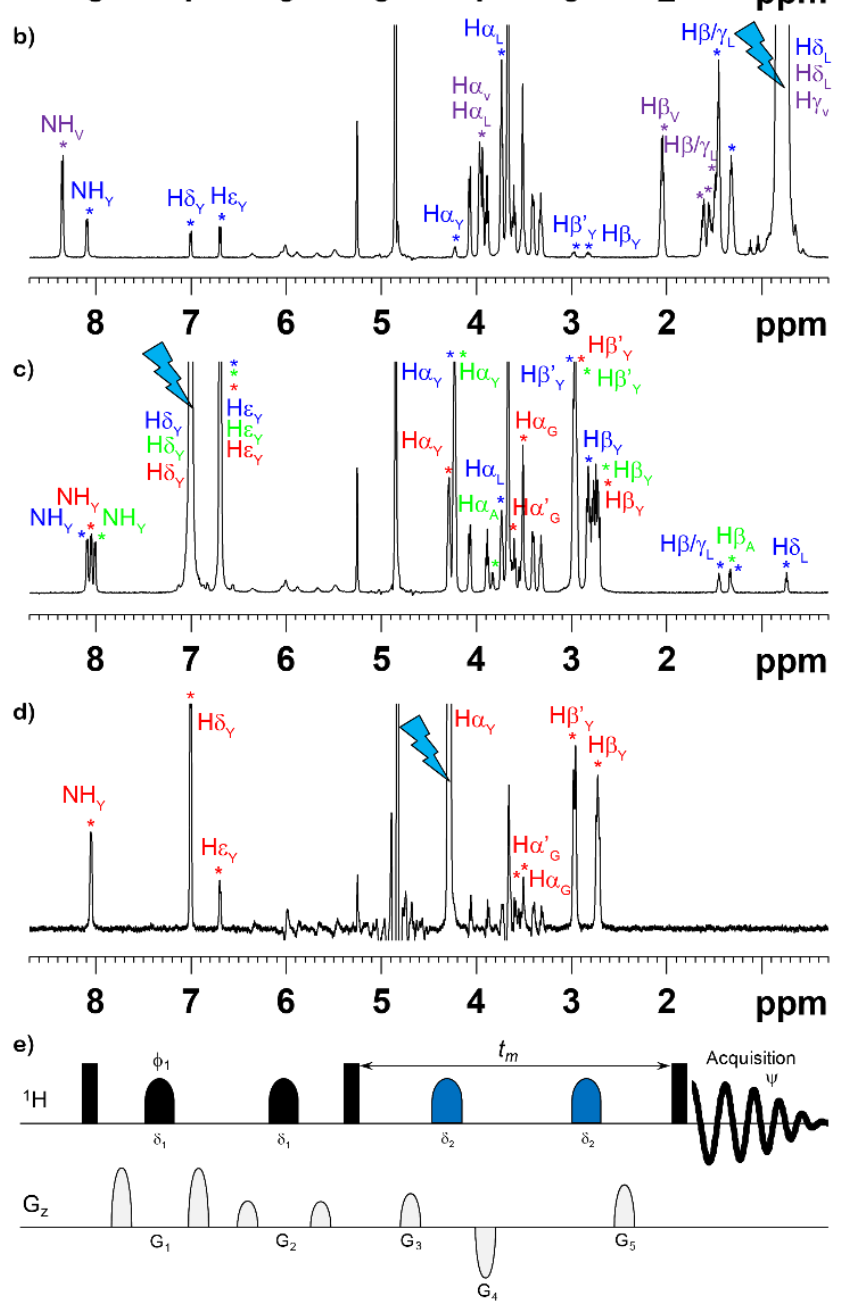

Fig. 3. Multiplet selective excitation $1 \mathrm{D}^{1} \mathrm{H}$ NOESY spectra of the dipeptide test mixture $(10 \mathrm{mM})$ dissolved in sucrose solution $(\mathrm{a}, \mathrm{b}$, c, d, $283 \mathrm{~K}), t_{m}=0.5 \mathrm{~s}$, at $600 \mathrm{MHz}\left({ }^{1} \mathrm{H}\right)$. The initial selective inversion pulses excite: a) the $\mathrm{NH}_{\mathrm{V}}(\mathrm{LV})$ proton resonance (experiment time (expt) $=134.65 \mathrm{~min}$ ); b) the
$\mathrm{H} \delta_{\mathrm{L}}(\mathrm{LY}) / \mathrm{H} \delta_{\mathrm{L}}(\mathrm{LV}) / \mathrm{H} \gamma_{\mathrm{V}}(\mathrm{LV})$ proton resonances (expt $\left.=33.67 \mathrm{~min}\right) ; \mathrm{c}$ ) the $\mathrm{H} \delta_{\mathrm{Y}}(\mathrm{LY}) / \mathrm{H} \delta_{\mathrm{Y}}(\mathrm{GY}) / \mathrm{H} \delta_{\mathrm{Y}}(\mathrm{AY})$ proton resonances (expt = $25.32 \mathrm{~min}$ ); d) the $H \alpha_{Y}(G Y)$ proton resonance (expt $=275.07 \mathrm{~min}$ ). e) Pulse sequence: $\varphi 1=x, y,-x,-y, \psi=x,-x$.

Another way to the individualization of dipeptide mixture which is less time-consuming than the acquisition of four suitable selective 1D NOESY spectra has been to focus on the close $\mathrm{NH}$ amide resonances as an initial source of magnetization and to resort to a $F_{1}$ band selective $F_{1}$ decoupled 2D NOESY experiment ${ }^{41,42}$ since these selected nuclei have their resonances in the same frequency band and they are not scalarly coupled together (see Figure 4). Carrying out this latter experiment in sucrose solution allows the assignment of all proton resonances of Leu-Val, Leu-Tyr, Gly-Tyr and Ala-Tyr by taking profit from spin diffusion acting during the mixing time.

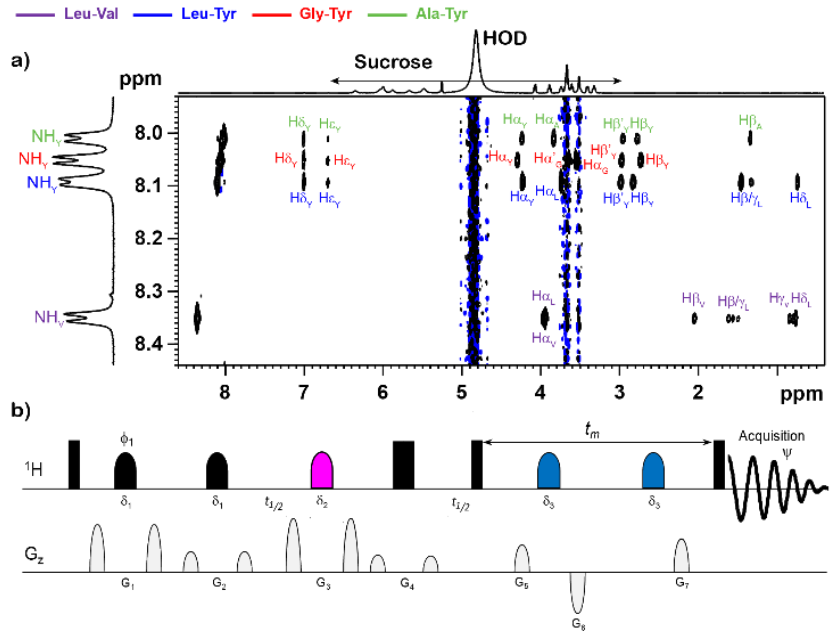

Fig. 4. a) $F_{1}$ band selective $F_{1}$ decoupled 2D NOESY spectrum of the dipeptide test mixture $(10 \mathrm{mM})$ in sucrose solution, at $283 \mathrm{~K}$, at 600 $\mathrm{MHz}\left({ }^{1} \mathrm{H}\right)$, (64 scans per $\mathrm{t}_{1}$ value, expt $\left.\left.=222.54 \mathrm{~min}, t_{m}=0.5 \mathrm{~s}\right) . \mathrm{b}\right)$ Pulse sequence: $\varphi 1=x, y,-x,-y, \psi=x,-x$. The initial selective $180^{\circ}$ pulses had a Gaussian shape and were applied to the four NH amide proton resonances.

The selective excitation of isolated proton resonances makes possible the individualization of each peptide within the mixture by taking advantage of spin diffusion. Nonetheless, it may happen in other complex mixtures that a component of interest does not present resolved proton resonances owing to strong spectral overlap. In such cases, the larger chemical shift dispersion of ${ }^{13} \mathrm{C}$ and ${ }^{15} \mathrm{~N}$ nuclei may prove to be helpful. By coupling the HSQC and NOESY experiments, a complete proton spectrum should be achieved for a molecule starting only from a single carbon or nitrogen resonance.

The $2 \mathrm{D}{ }^{1} \mathrm{H}-{ }^{13} \mathrm{C}$ and ${ }^{1} \mathrm{H}-{ }^{15} \mathrm{~N}$ HSQC-NOESY spectra of the dipeptide test mixture have been acquired at $283 \mathrm{~K}$ in sucrose solution (Figure S-5 in ESI and Figure 5). Under these viscous conditions, all protons of each dipeptide of the mixture are able to propagate its own magnetization with all other protons and protonated carbons or protonated nitrogen by spin diffusion. A suitable selection of horizontal rows through carbon resonances at 18.14 and 38.08 ppm 
(Ala-Tyr), 22.64 and 37.66 ppm (Leu-Tyr), 31.43 and 53.64 ppm (Leu-Val) and $38.11 \mathrm{ppm}$ (Gly-Tyr) allows the extraction of the four complete proton spectra respectively corresponding to Ala-Tyr, LeuTyr, Leu-Val and Gly-Tyr. These four spectra have been compared to the conventional $1 \mathrm{D}{ }^{1} \mathrm{H}$ spectra (Figures S-5b, b', S-5c, c', S-5d, d' and $\mathrm{S}-5 \mathrm{e}, \mathrm{e}^{\prime}$ in ESI) and they logically reveal similar peak patterns. In the same way, an appropriate selection of four horizontal slices through nitrogen resonances at 123.93 (Ala-Tyr), 124.49 (Gly-Tyr), 126.01 (Leu-Tyr), and $126.91 \mathrm{ppm}$ (Leu-Val) allows to produce four complete ${ }^{1} \mathrm{H}$ spectra respectively corresponding to Ala-Tyr, Gly-Tyr, Leu-Tyr, and Leu-Val. These four spectra present resonance patterns similar to those of the conventional $1 \mathrm{D}^{1} \mathrm{H}$ spectra as well (Figures $5 b, b^{\prime}, 5 c, c^{\prime}, 5 d, d^{\prime}$ and $5 e, e^{\prime}$ ). Interestingly, another way is possible to individualize the compounds of the mixture by considering appropriate vertical slices from the $2 \mathrm{D}{ }^{1} \mathrm{H}-{ }^{13} \mathrm{C}$ HSQCNOESY (Figure S-5). In this case, the resulting spectra should reveal all the protonated carbons of the four dipeptides in the mixture. By the way, Figure S-5 ( $d^{\prime \prime}, c^{\prime \prime}, e^{\prime \prime}$ and $\left.b^{\prime \prime}\right)$ presents the four protonated carbon spectra obtained by extracting the column of amide protons at $8.35,8.09,8.05$ and $8.01 \mathrm{ppm}$, respectively corresponding to LeuVal, Leu-Tyr, Gly-Tyr, and Ala-Tyr. The ability to extract all the protonated carbon chemical shifts for an individual component in a mixture may turn out to be a very convenient tool in the structure assignment of molecules within mixtures.

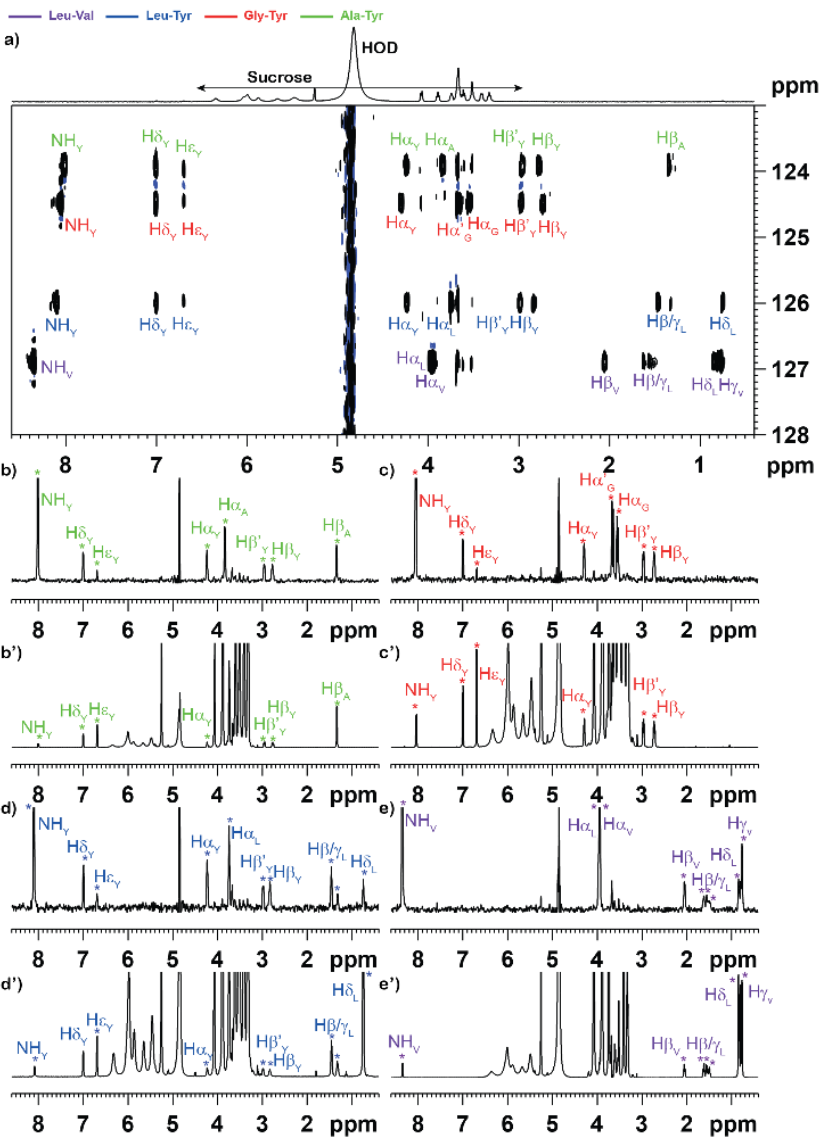

Fig. 5. a) $2 \mathrm{D}{ }^{1} \mathrm{H}_{-}{ }^{15} \mathrm{~N}$ HSQC-NOESY spectrum of the dipeptide test mixture $(20 \mathrm{mM})$ dissolved in sucrose solution, at $283 \mathrm{~K}, t_{m}=0.5 \mathrm{~s}$, with solvent multiple presaturation, at $600 \mathrm{MHz}\left({ }^{1} \mathrm{H}\right)$. Comparison of four ${ }^{1} \mathrm{H}$ horizontal slices extracted from the $2 \mathrm{D}^{1} \mathrm{H}-{ }^{15} \mathrm{~N}$ HSQC-NOESY at 123.93 (b, b', Ala-Tyr, green row), 124.49 (c, c', Gly-Tyr, red row), 126.01 (d, d', Leu-Tyr, blue row), and 126.91 ppm (e, e', Leu-Val, purple row) with the conventional 1D proton spectra of each pure dipeptide dissolved $(20 \mathrm{mM})$ in sucrose solution, at $283 \mathrm{~K}$, at 600 $\mathrm{MHz}\left({ }^{1} \mathrm{H}\right)$.

\section{Leu-Val, Leu-Tyr, Gly-Tyr and Ala-Tyr mixture in agarose gel}

As previously published, ${ }^{36,37}$ owing to the active transverse relaxation of agarose gel, all the proton resonances from agarose are remarkably not visible (see Figure 6). This aspect is probably the main advantage of using this viscous medium in the individualization of components within polar mixtures because that makes easier the solvent suppression.

We also determined the temperature that provides the best compromise between spin diffusion and spectral resolution by means of usual NOESY experiments with water suppression using excitation sculpting (see Figure S-6 in ESI). The optimized temperature of $273 \mathrm{~K}$ enables to detect as much as possible negative NOE enhancements (positive NOESY cross peaks) due to the molecular tumbling slowdown. The use of viscous agarose gel yields full intramolecular magnetization transfer through spin diffusion, over distances of $>14 \AA$ for Leu-Tyr, Gly-Tyr and Ala-Tyr owing to the stiffness of the aromatic moiety. In comparison, the NOESY spectrum acquired in water at $298 \mathrm{~K}$ presents less NOE cross peaks of opposite sign (positive NOE enhancements, fast molecular tumbling, see Figure $2 \mathrm{~b}$ ). However, the remaining flexibility of LeuVal peptide in agarose gel at $273 \mathrm{~K}$ prevents the observation of full magnetization transfer over the entire molecule by means of spin diffusion. Only positive NOE enhancements are detected. Nonetheless, under sufficiently viscous conditions, the grouping of proton resonances of Tyr-based dipeptides together is reachable, thus allowing the individualization of these three Tyr-based mixture components since the chemical shift pattern of each dipeptide is predictable. By subtraction, the proton resonance pattern of LeuVal is then deduced.

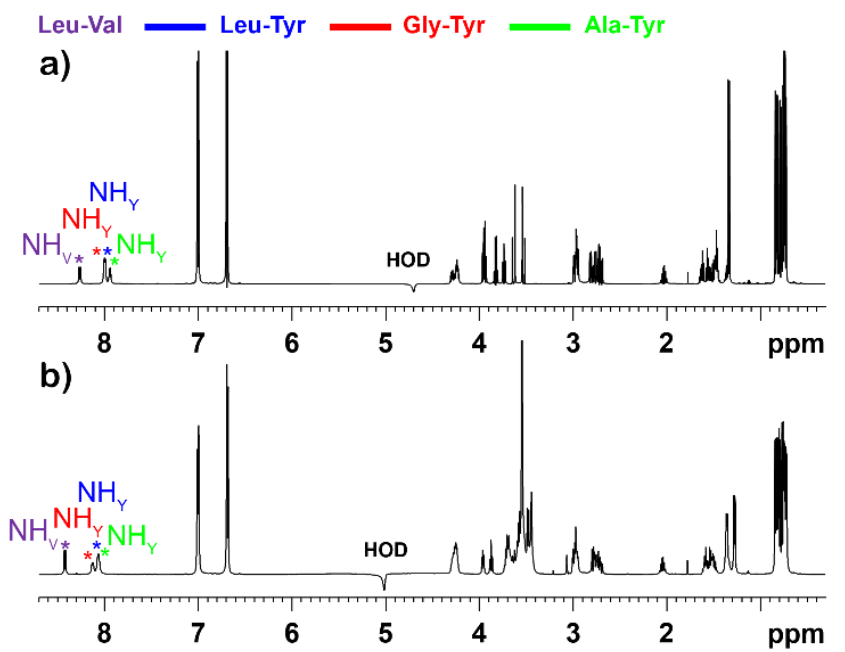

Fig. 6. $1 \mathrm{D}{ }^{1} \mathrm{H}$ spectra of the dipeptide test mixture $(10 \mathrm{mM})$ dissolved a) in $\mathrm{H}_{2} \mathrm{O} / \mathrm{D}_{2} \mathrm{O}(9: 1, \mathrm{v} / \mathrm{v})$, at $298 \mathrm{~K}$, at $600 \mathrm{MHz}\left({ }^{1} \mathrm{H}\right)$ and b) 
in agarose gel $1 \%$, at $273 \mathrm{~K}$, at $600 \mathrm{MHz}\left({ }^{1} \mathrm{H}\right)$ with water suppression using excitation sculpting.

We sought to highlight the discrepancy of the molecular behaviour of the four dipeptides in agarose gel by only collecting the resonances of interest during the signal acquisition for accessing to supplementary structural information. This approach may turn out to be pertinent in case of proton overlapping especially for complex mixtures. Selectively exciting one suitable set of proton resonances illustrates this approach. By the way, we reused the 1D selective NOESY experiment previously described in this work. The only one HOD signal of agarose gel arising from longitudinal relaxation during the mixing time is more easily suppressed than the multiple ones of sucrose. Figure 7 illustrates that all Tyr-based dipeptides are differentiated by spin diffusion in agarose gel by means of an appropriate set of selectively excited proton resonances. Indeed, the selective excitation of $\mathrm{CH}_{3 \beta}$ at $\delta 1.27$ in agarose gel, shows a magnetization exchange exclusively with the protons of the Ala-Tyr dipeptide (Figure 7a). The selective excitation of the aromatic $\mathrm{H} \delta / \mathrm{H} \varepsilon$ protons of Leu-Tyr, Gly-Tyr and Ala-Tyr at $\delta$ 7.00 reveals all the proton resonances of Leu-Tyr, Gly-Tyr and AlaTyr (Figure 7b). The selective excitation of the side chain $\mathrm{H} \delta$ and $\mathrm{Hr}$ protons (between 0.7 and $0.9 \mathrm{ppm}$ ) displays a magnetization exchange with all protons of the two Leu-Val and Leu-Tyr dipeptides (Figure 7c). Due to a different molecular tumbling rate, the NOE enhancements are negative (active spin diffusion, positive peaks) for Leu-Tyr and positive for Leu-Val (negative peaks). As a result, the distinction of the two proton patterns is immediate by considering the change in the sign of the NOE peaks. By comparison with the 1D NOESY spectra in Figures $7 a, 7 b$ and $7 c$, a complete proton assignment of Gly-Tyr is accessible.

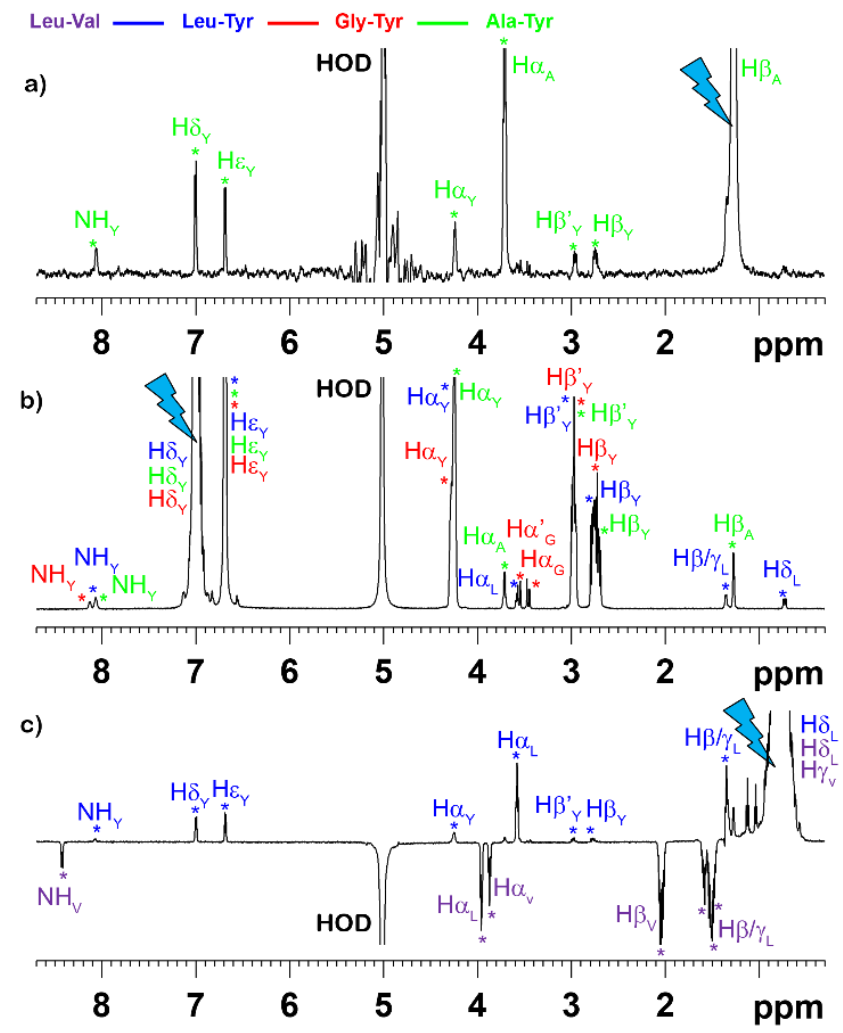

Fig. 7. Multiplet selective excitation $1 \mathrm{D}{ }^{1} \mathrm{H}$ NOESY spectra of the dipeptide test mixture $(10 \mathrm{mM})$ dissolved in agarose gel $1 \%(\mathrm{a}, \mathrm{b}, \mathrm{c}$, $273 \mathrm{~K}), t_{m}=0.5 \mathrm{~s}$, at $600 \mathrm{MHz}\left({ }^{1} \mathrm{H}\right)$. The initial selective inversion pulses excite: a) the $H \beta_{Y}(A Y)$ proton resonance (expt = 186,25 min); b) the $H \delta_{Y}(L Y) / H \delta_{Y}(G Y) / H \delta_{Y}(A Y)$ proton resonances (expt $=67,25$ min); c) the $H \delta_{\mathrm{L}}(\mathrm{LY}) / \mathrm{H} \delta_{\mathrm{L}}(\mathrm{LV}) / H \gamma_{\mathrm{V}}(\mathrm{LV})$ proton resonances (expt $=$ $67,08 \mathrm{~min}$ ); Pulse sequence: $\varphi 1=x, y,-x,-y, \psi=x,-x$ (see Figure $3 e)$.

\section{Experimental}

Chemical reagents. $\mathrm{D}_{2} \mathrm{O}$ was purchased from Eurisotop (Gif-surYvette, France). Leu-Val, Leu-Tyr, Gly-Tyr and Ala-Tyr were purchased from $\mathrm{TCl}$ Europe (Zwijndrecht, Belgium). Agarose/TBE blend powder $1 \%$ was purchased from SigmaAldrich (Saint-Quentin-Fallavier, France). All peptides had 95\% or higher purity and were dissolved at a concentration from 10 to $20 \mathrm{mM}$ in sucrose $/ \mathrm{H}_{2} \mathrm{O}\left(5: 5, \mathrm{w} / \mathrm{w}+10 \% \mathrm{D}_{2} \mathrm{O}, \mathrm{v} / \mathrm{v}\right)$, and agarose gel $1 \% / \mathrm{D}_{2} \mathrm{O}(9: 1, \mathrm{v} / \mathrm{v})$.

NMR Spectroscopy: All the NMR experiments on the dipeptide mixture were performed on a Bruker Avance AVIII-600 NMR spectrometer equipped with a $5 \mathrm{~mm} \mathrm{TCl}$ cryoprobe using the Bruker TOPSPIN Software (Rheinstetten, Germany). Static field gradient pulses were generated by a $10 \mathrm{~A}$ amplifier, so that the sample is submitted to a nominal $0.613 \mathrm{Tm}^{-1}$ gradient. Gradient pulses were followed by a $200 \mu \mathrm{s}$ recovery delay. Temperature control was performed using a Bruker variable temperature (BVT) unit in combination with a Bruker cooling unit (BCU-05) to provide chilled air.

Dipeptide mixture spectra were calibrated so that the tyrosine $\mathrm{H} \alpha$ proton and $\mathrm{C} \alpha$ carbon resonances appeared respectively at 7.00 and 132.00 ppm. Additional NMR data acquisition and processing parameters for Figure 1 up to Figure 7 are described in the Electronic Supplementary Information S-1 to s-6.

\section{Conclusions}

We have established for the first time that the use of sucrose solution and agarose gel makes the resolution of polar components within complex mixtures possible, by taking advantage of NMR spin diffusion. We have pointed out that using sucrose blends as viscous binary solvent presents valuable advantages compared to agarose gel: spin diffusion is active in a wider temperature range, NMR sample preparation (no heating) is compatible with thermally fragile compounds and user-friendly, even though agarose gel presents the considerable benefit of not producing intense residual proton solvent due to active transverse relaxation that is the major drawback of the use of non-deuterated sucrose solution. Mid-sized molecules will only require a small amount of sucrose in water whereas smaller molecules will require more sucrose until 50\% $(w / w)$ for driving spin diffusion from room temperature to sub-zero 
temperature. Agarose gel may be a suitable alternative to the use of sucrose solution for the study of small rigid or mid-sized compounds in mixture presenting quite long correlation times for which spin diffusion should be more active than that of very small and flexible molecules.

The component individualization within a Leu-Val, Leu-Tyr, GlyTyr and Ala-Tyr mixture was respectively achieved at $283 \mathrm{~K}$ and 273 $\mathrm{K}$ in sucrose solution and agarose gel by means of selective $1 \mathrm{D}, 2 \mathrm{D}$ ${ }^{1} \mathrm{H}-{ }^{1} \mathrm{H}$ NOESY. ${ }^{1} \mathrm{H}-{ }^{13} \mathrm{C}$ and ${ }^{1} \mathrm{H}-{ }^{15} \mathrm{~N}$ HSQC-NOESY experiments were implemented for sucrose solution since ${ }^{13} \mathrm{C}$ and ${ }^{15} \mathrm{~N}$ nuclei were considered as chemical shift markers that increase the spectrum readability, at the price of a lower sensitivity caused by their low natural abundance.

Future investigations will deal the study of other polar mixtures of medium-sized molecules for assessing spin diffusion power of agarose gel and of the solute-solvent interaction.

\section{Conflicts of interest}

The authors declare no conflict of interest.

\section{Acknowledgements}

We wish to thank CNRS, Conseil Regional Champagne Ardenne, Conseil General de la Marne and the EU-programme FEDER to the PIAneT CPER project is gratefully acknowledged.

\section{Notes and references}

1.

114, 3139-3141.

K. F. Morris and C. S. Johnson, J. Am. Chem. Soc., 1993 115, 4291-4299.

3. K. F. Morris, P. Stilbs and C. S. Johnson, Anal. Chem., 1994 66, 211-215.

4. S. Viel, F. Ziarelli and S. Caldarelli, Proc. Natl. Acad. Sci. USA, 2003, 100, 9696-9698.

5. G. Pages, C. Delaurent and S. Caldarelli, Angew. Chem. Int Ed. , 2006, 45, 5950-5953.

6. G. Pages, C. Delaurent and S. Caldarelli, Anal. Chem. 2006, 78, 561-566.

7. S. Caldarelli, Magn. Reson. Chem., 2007, 45, S48-S55

8. C. Carrara, S. Viel, F. Ziarelli, G. Excoffier, C. Delaurent and S. Caldarelli, J. Magn. Reson., 2008, 194, 303-306.

9. M. E. Zielinski and K. F. Morris, Magn. Reson. Chem., 2008, 47, 53-56

10. R. Evans, S. Haiber, M. Nilsson and G. A. Morris, Anal. Chem., 2009, 81, 4548-4550.

11. M. E. Zielinski and K. F. Morris, Magn. Reson. Chem. 2009, 47, 53-56.

12. J. S. Kavakka, V. Parviainen, K. Wähälä, I. Kilpeläinen and S. Heikkinen, Magn. Reson. Chem., 2010, 48, 777-781.

13. A. K. Rogerson, J. A. Aguilar, M. Nilsson and G. A. Morris, Chem. Commun., 2011, 47, 7063-7064.

14. C. Pemberton, R. Hoffman, A. Aserin and N. Garti, J. Magn. Reson., 2011, 208, 262-269.
15. J. C. Hoch, M. W. Maciejewski and B. Filipovic, J. Magn Reson., 2008, 193, 317-320.

16. M. Mobli, M. W. Maciejewski, A. D. Schuyler, A. S. Stern and J. C. Hoch, Phys. Chem. Chem. Phys., 2012, 14, 10835 10843.

17. N. H. Meyer and K. Zangger, Angew. Chem. Int. Ed., 2013, 52, 7143-7146.

18. K. Kazimierczuk and V. Orekhov, Magn. Reson. Chem., 2015, 53, 921-926.

19. C. Papaemmanouil, C. G. Tsiafoulis, D. Alivertis, O. Tzamaloukas, D. Miltiadou, A. G. Tzakos and I. P. Gerothanassis, J. Agric. Food. Chem., 2015, 63, 5381-5387.

20. K. Zangger, Prog. Nucl. Magn. Reson. Spectrosc., 2015, 8687, 1-20.

21. G. D. Poggetto, L. Castañar, G. A. Morris and M. Nilsson, RSC Adv., 2016, 6, 100063-100066.

22.

A. Gierer and K. Wirtz, Zeitschrift Für Naturforschung Section A, 1953, 8, 532-538.

23. M. P. Williamson and D. H. Williams, J. Chem. Soc., Chem Commun., 1981, 4, 165-166.

24. A. J. Simpson, G. Woods and O. Mehrzad, Anal. Chem. 2008, 80, 186-194.

25. H. Farooq, R. Soong, D. Courtier-Murias, C. Anklin and A. Simpson, Anal. Chem., 2012, 84, 6759-6766.

26. P. Lameiras, L. Boudesocque, Z. Mouloungui, J. H. Renault, J. M. Wieruszeski, G. Lippens and J. M. Nuzillard, J. Magn. Reson., 2011, 212, 161-168.

27. P. Lameiras and J.-M. Nuzillard, Anal. Chem., 2016, 88 4508-4515.

28. P. Lameiras, S. Patis, J. Jakhlal, S. Castex, P. Clivio and J. M. Nuzillard, Chem. Eur. J., 2017, 23, 4923-4928.

29. V. R. N. Telis, J. Telis-Romero, H. B. Mazzotti and A. L. Gabas, Int. J. Food Prop., 2007, 10, 185-195.

30. M. P. Longinotti and H. R. Corti, J. Phys. Chem. Ref. Data 2008, 37, 1503-1515.

31. C. H. Cho, J. Urquidi, S. Singh and G. W. Robinson, J. Phys. Chem. B, 1999, 103, 1991-1994.

32

M. O. Sonnati, S. Amigoni, E. P. Taffin De Givenchy, T. Darmanin, O. Choulet and F. Guittard, Green Chem., 2013, 15, 283-306.

33. E. Bismuto and G. Irace, FEBS Lett., 2001, 509, 476-480

34. M. Ausubel, R. Brent, R. E. Kingston, D. D. Moore, J. G. Seidman, J. A. Smith and S. K., Mol. Reprod. Dev., 1989, 1, 146-146.

35. R. Kim, H. Yokota and S. H. Kim, Anal. Biochem., 2000, 282, 147-149.

36. A. M. Spring and M. W. Germann, Anal. Biochem., 2012, 427, 79-81.

37. A. Pastore, S. Salvadori and P. A. Temussi, J. Pept. Sci. 2007, 13, 342-347.

38. K. Stott, J. Stonehouse, J. Keeler, T.-L. Hwang and A. J. Shaka, J. Am. Chem. Soc., 1995, 117, 4199-4200.

39. K. Wüthrich, 1986, New York: Wiley.

40. K. Stott, J. Keeler, Q. N. Van and A. J. Shaka, J. Magn. Reson., 1997, 125, 302-324.

41. R. Brüschweiler, C. Griesinger, O. W. Sørensen and R. R. Ernst, J. Magn. Reson., 1988, 78, 178-185.

42. B. Plainchont, A. Martinez, S. Tisse, J. P. Bouillon, J. M. Wieruszeski, G. Lippens, D. Jeannerat and J. M. Nuzillard, J. Magn. Reson., 2010, 206, 68-73. 